\title{
MAPEAMENTO DO MDA E HABILIDADES DO JOGADOR NO GRÁFICO DA TEORIA DO FLUXO
}

\author{
Guilherme Zaffari \\ Universidade Federal do Paraná - UFPR \\ guizaffari@gmail.com \\ André Luiz Battaiola \\ Universidade Federal do Paraná - UFPR \\ ufpr.design.profe.albattaiola@gmail.com
}

Resumo: Em anos recentes, uma variedade de teorias a respeito do Design de Jogos Digitais vem sendo publicadas. Suas intenções variam: algumas procuram descobrir o que torna um jogo "divertido", outros trabalham os diferentes componentes que os constroem. Um problema notado é que esses trabalhos estão fazendo propostas similares, entretanto separadamente. A intenção deste artigo, por conseguinte, é estudar algumas dessas teorias e mapeá-las, para que possam se relacionar e, assim, aprimorar o conhecimento para projetar um jogo de qualidade. A base para essas relações está no frameowrk MDA para o design de jogos, criado por Robin Hunicke, Marc LeBlanc, e Robert Zubek. As teorias associadas compreendem a Teoria do Fluxo de Mihalyi Csikszentmihalyi, até as diversas técnicas de criação e análise de jogos de Aki Järvinen. O resultado da combinação mostra que o gráfico da teoria do fluxo suporta as proposições estudadas e ajuda a reforçar o objetivo do modelo MDA, de unificação de conceitos.

Palavras-chave: MDA; Teoria do Fluxo; Habilidades do Jogador.

Abstract: In recent years, various theories regarding Game Design have been published. Their intentions varies: some beseech to discover what makes a game "fun", others regards the many concepts that form them. An issue noticed is that these works are making similar breakthroughs, and presented separately. Therefore, the intention of this paper is to study some of these theories and map them, so that they can be related, thus making the understating of designing a successful and fun game predictable. The basis for these relations is the MDA framework for game design, created by Robin Hunicke, Marc LeBlanc, and Robert Zubek. The theories being associated ranges from Mihaly Csikszentmihalyi's Flow Theory, to Aki Järvinen's many theories for studying and analyzing games. The combination results shows that the flow theory chart can withstand the 
studied propositions and that it helps reinforce the MDA framework objective of integrating concepts.

Keywords: MDA; Flow theory; player abilities.

\section{INTRODUÇÃO}

O design de jogos digitais é um tema que, na academia, tem angariado bastante interesse. Somente no SB Games de 2013 (a maior conferência do Brasil sobre o tema games) 54 artigos foram publicados na seção "Arte e Design", sendo que 19 deles abordam especificamente à técnicas de desenvolvimento, metodologias e o relato do projeto de jogos digitais (2013, web). Internacionalmente, por exemplo, a revista Game Developer Magazine apresenta ao todo, no mesmo ano, 104 artigos publicados com conteúdo referentes ao game design (2013, www.gdcvault.com/gdmag).

A pluralidade de publicações demonstra que existe um interesse, por parte da academia, em estudar o assunto. Essa questão pode ser explicada por diversas razões, algumas apontadas pela associação americana, ESA - Entertainment Software Association. Nos seus estudos de 2012, consta que 50\% dos americanos jogam videogames, $45 \%$ dos jogadores são mulheres e $51 \%$ das casas americanas possuem uma plataforma de jogos digitais dedicada, se não duas. A questão fenomenológica da expansão da indústria dos jogos digitais é o que desperta a curiosidade de pesquisadores.

O estudo do design de jogos digitais, por parte dos estudiosos, produziu trabalhos acadêmicos que buscam agilizar sua produção conceitual, facilitar a compreensão entre as partes envolvidas no seu desenvolvimento e descobrir o que faz um jogo ser "divertido" ou bem sucedido, sendo o modelo MDA, de Hunicke, LeBlanc e Zubek (2004) é um exemplo de resultado desses estudos. Entretanto essas teorias encontradas não se inter-relacionam, apesar de possuírem pontos em comum. Neste artigo é formulada uma proposta de mapeamento do modelo citado, além da teoria das habilidades dos jogadores de Järvinen (2008), no gráfico de Csikszentmihalyi (1991) sobre a teoria do fluxo. Essa associação será feita mediante revisão bibliográfica das teorias, as quais serão expostas nos itens à seguir.

\section{O MODELO MDA: Mechanics, Dynamics and Aesthetics}

O modelo MDA foi um projeto desenvolvido e ensinado como parte de um workshop na Game Developer Conference de San José, nos anos de 2001 e 2004 pelos seus autores, Hunicke, LeBlanc e Zubek (2004). A sigla significa Mechanics, Dynamics e Aesthetics que, quando traduzidas para o português, são Mecânicas, Dinâmicas e Estética. A proposta dos autores foi criar um modelo de design que respeitasse uma metodologia projetual, pois, como afirmam os autores, "Todos os artefatos são criados a partir de alguma metodologia de design". A intenção dos autores é de fazer com que o diálogo entre as diferentes partes envolvidas possam se comunicar melhor durante 0 desenvolvimento de jogos digitais, alinhando conceitos entre designers.

Segundo Hunicke et al. (2004), o MDA formaliza em um diagrama que o consumo de jogos digitais é feito primeiro pelas regras, depois pela interação com seu 
sistema, o que leva à diversão. Eles ainda fazem uma associação onde Mecânicas = Regras, Dinâmicas $=$ Sistema e Estética $=$ Diversão. 0 esquema desse artigo pode ser observado na figura 1, à seguir:

O MDA formaliza a estrutura de consumo de um jogo:

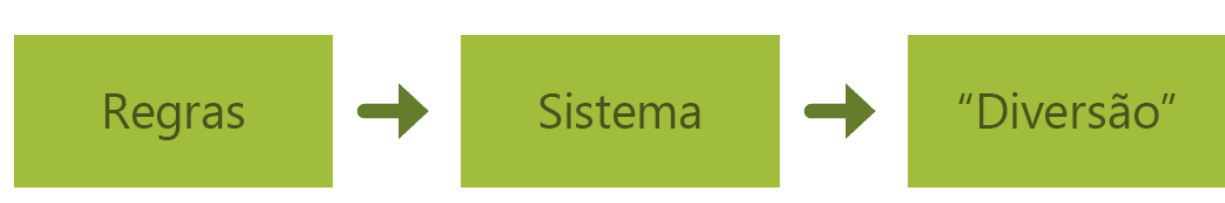

... e faz o paralelo com suas partes no design:

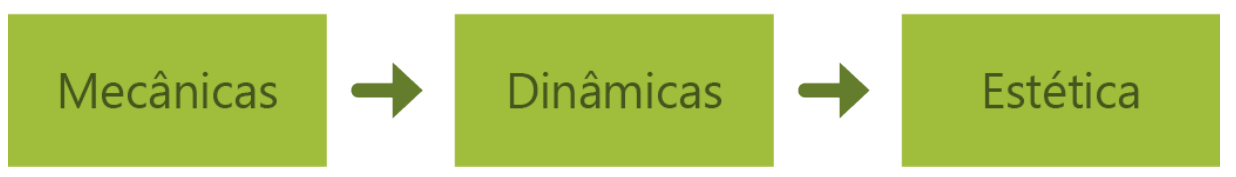

Figura 1 - Esquematização da formalização do design de jogos.

Fonte: Adaptado de Hunicke et al. (2003).

Mais detalhadamente, Mecânicas, Dinâmicas e Estética são descritas, por Hunicke et al. (2004) da seguinte forma:

- Mecânicas: Descreve os componentes essenciais do jogo em termos de representação de dados numéricos e algoritmos.

- Dinâmicas: Descreve o comportamento das mecânicas em tempo real, agindo sobre as entradas e saídas de dados do jogador no decorrer de uma partida.

- Estética: Descreve a resposta emocional desejada, invocada no jogador, quando ele interage com o sistema do jogo.

Os autores ainda ressaltam que o desenvolvimento de jogos digitais é uma "via de duas mãos", onde jogador e designer observam o artefato (o jogo digital) sob diferentes perspectivas. Observa-se o diagrama abaixo representado, sem alterações do material de fonte, na figura 2 : 


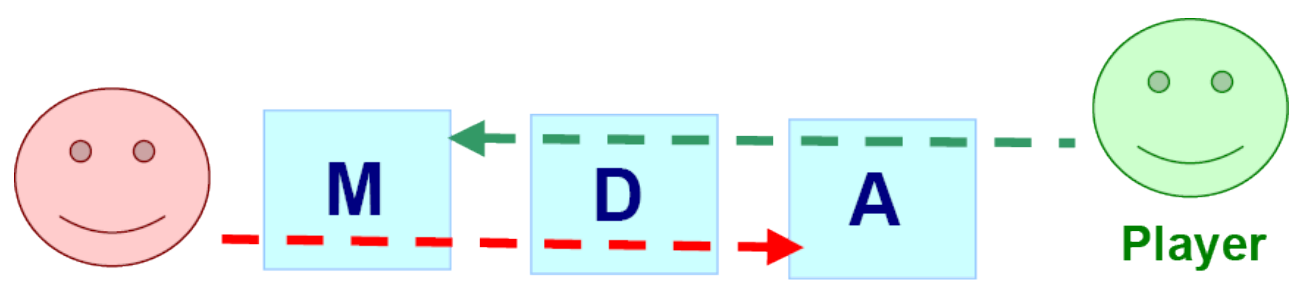

Designer

Figura 2 - Diagrama explicando a perspectiva de observação de jogador e design.

Fonte: Hunicke et al. (2004)

Ressaltado que a perspectiva do jogador difere do desenvolvedor, os autores teorizam o que faz um jogo divertido. Para fazê-lo, eles explicam que seu conceito de estética consiste de oito elementos:

1. Sensação: Jogo como prazer dos sentidos.

2. Fantasia: Jogo como faz-de-conta

3. Narrativa: Jogo como drama

4. Desafio: Jogo como corrida de obstáculos

5. Sociedade: Jogo como modelo social

6. Descoberta: Jogo como território inexplorado

7. Expressão: Jogo como autoconhecimento

8. Submissão: Jogo como passatempo

O design do jogo, segundo Hunicke et al. (2004), busca: "múltiplos objetivos estéticos, em graus variados". Eles citam que ao seguir esse vocabulário de experiências estéticas, pode-se começar o desenvolvimento de dinâmicas e mecânicas. Para exemplificar este uso, os autores se referem a um jogo de adivinhação com mímica, colocando em prática o conceito de Desafio. Os jogadores devem estar emocionalmente engajados com a atividade a fim de torná-la genuinamente desafiante. Esta experiência, segundo Hunicke et al. (2004) é o que faz o jogo ser "divertido".

Para melhor explicitar seu modelo, os autores apresentam formas de se tratar dinâmicas aplicando os conceitos estéticos, mesclando-os com mecânicas que prevêem, nos usuários, as experiências desejáveis:

- Desafio: Pressão do tempo e jogada do adversário.

- Sociedade: Divisão de recursos, troca de informações e elaboração de estratégias para alcançar um objetivo maior, que não seria possível sozinho. 
- Expressão: Customização de personagens, interação com o ambiente do jogo (e seus habitantes).

- Narrativa: Roteiro com tensão dramática bem trabalhada. Boa estrutura e desfecho emocionantes.

Por fim, os autores ainda fundamentam que mecânicas são: "as várias ações, comportamentos e mecanismos de controle adquiridos pelo jogador dentro do contexto do jogo. Ao lado do conteúdo do jogo, as mecânicas dão subsídio às dinâmicas da experiência de jogo em geral." Hunicke et al. (2004). Pode-se concluir, portanto, que em um jogo de xadrez, por exemplo, as mecânicas de movimentação das peças e de captura das mesmas pode gerar dinâmicas de estratégias que, por sua vez, incitam a experiência estética de Desafio entre os jogadores.

\section{A TEORIA DO FLUXO}

A experiência estética teorizada por Hunicke et al. (2004) e o conceito de "divertido" pode ser explicada, também, pela teoria de Mihaly Csikszentmihaly (1991), a Teoria do Fluxo. Nela, ele afirma que a diversão é fruto do desempenho em uma determinada atividade: "...o que os mantinham motivados era a qualidade da experiência que sentiam quando envolvidos na atividade.". O autor ainda relata que, em seus estudos, as pessoas procuram esforços arriscados, difíceis, com um certo grau de "novidade". A situação que esses indivíduos se encontram é o que ele chama de estado de fluxo. Basicamente, o que Csikszentmihalyi (1990) diz é que a parte interessante para o ser humano não é a atividade a desempenhar, ou a recompensa por fazê-la (apesar de serem partes fundamentais para o estado de fluxo), mas sim a experiência da tarefa em si que é o cerne da diversão.

No seu livro, o autor descreve que, para uma atividade ser divertida, ela precisa necessariamente possuir os seguintes nove elementos:

1. Existem objetivos bem definidos a cada passo da jornada: Csikszentmihalyi (1990) explica que tarefas corriqueiras, comuns à rotina não possuem propósito pessoal, ou são contraditórias. Quando estamos em fluxo, entretanto, sabemos exatamente o que temos que fazer. Em uma partida de xadrez cada movimento de peça segue uma intenção geral, capturar o rei;

2. Ocorre um retorno imediato e suficiente para cada ação de um indivíduo: Csikszentmihalyi (1990) mostra que, diferente das situações normais, quando em fluxo temos noção de o que fazemos está certo. Em uma partida de xadrez, sabemos que estamos jogando bem, por exemplo, quando temos mais peças do que o adversário no tabuleiro;

3. Ocorre um equilíbrio entre desafios e habilidades: Segundo Csikszentmihalyi (1990), entramos em fluxo quando uma conversa, ou uma atividade que engajamos, desafia-nos intelectualmente à altura de nossas habilidades. Uma partida de xadrez, por exemplo os jogadores se mantém no limiar entre o tédio e a ansiedade, devido à constante pressão adversária;

4. Ações e prontidão estão combinadas: Durante o estado de fluxo, Csikszentmihalyi (1990) escreve que nossos pensamentos estão 
completamente focados na atividade, pois existe constante retorno e objetivos claramente definidos.

5. Dimensões são excluídas da consciência: Quando em fluxo, Csikszentmihalyi (1990) comenta que o estado de concentração do indivíduo é tal que a noção espacial desaparece, dando lugar (no processamento cognitivo) apenas para a atividade.

6. Não existe preocupação em falhar: Devido ao estado de concentração extrema, do estado de fluxo, Csikszentmihalyi (1990) ressalta que a preocupação em falhar não é relevante para o cérebro do indivíduo. Apenas a atividade importa.

7. A autoconsciência desaparece: $O$ estado de fluxo, segundo Csikszentmihalyi (1990) nos faz perder a noção da nossa própria aparência perante os outros. Estamos envolvidos demais com a atividade para preocupar-nos com trivialidades como trato social, por exemplo.

8. A percepção do tempo se torna distorcida: Em estado de fluxo, um indivíduo pode perceber que o tempo "corre" mais rapidamente, ou mais devagar que o normal. Segundo Csikszentmihalyi (1990) nossa preocupação com a passagem do tempo não é mais relevante à nossa atenção.

9. A atividade se torna um propósito dentro de si mesma: $O$ autor explica que, quando todos estes elementos combinados estiverem presentes em uma atividade, ela se torna autotélica, ou seja, fazê-la é seu próprio propósito.

Para explicar como se chega a este estado de fluxo, o autor sugere um gráfico cartesiano composto por duas variáveis onde, no eixo $X$ se encontra o nível das habilidades do indivíduo e no eixo $\mathrm{Y}$, a complexidade do desafio. Abaixo, a representação do gráfico (figura 3), como teorizado por Csikszentmihalyi (1990):

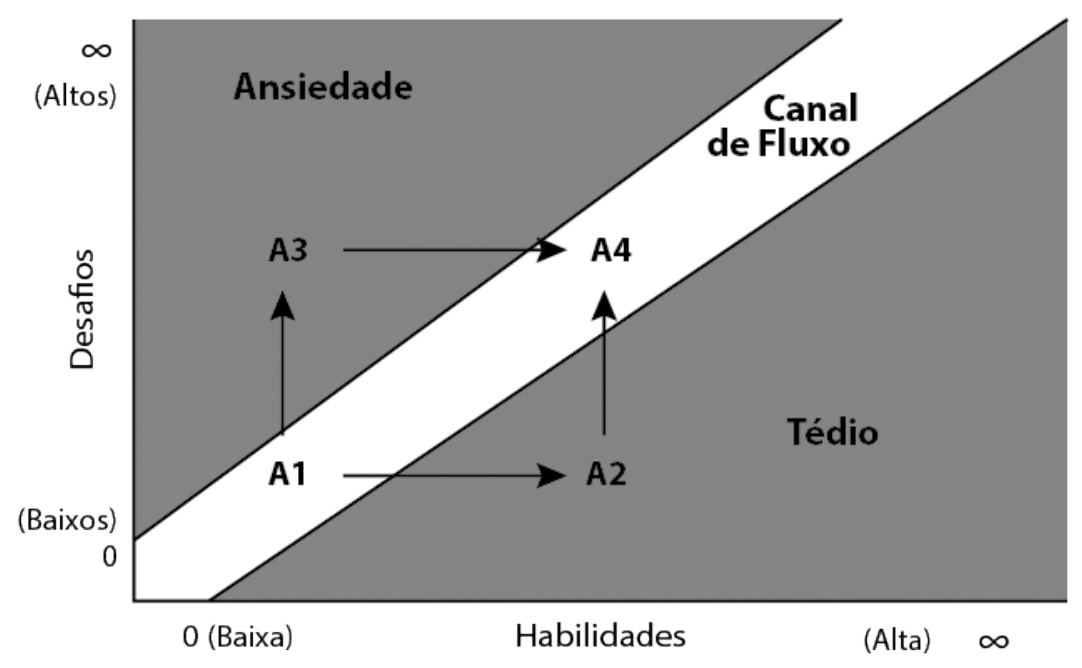

Figura 3 - Gráfico da teoria do fluxo.

Fonte: Adaptado de Csikszentmihalyi (1990). 
Para explicar o gráfico, Csikszentmihalyi (1990) fornece o seguinte exemplo: A letra " $A$ " representa Alex, um garoto aprendendo a jogar tênis. Na primeira atividade dele (A1), o nível de habilidade com o esporte é pequeno. Ele joga uma bola contra uma parede e fica rebatendo-a. Ele está em fluxo.

Depois de um tempo, o menino adquire um nível maior de habilidade, levandoo à posição $A 2$, no gráfico. Isso significa que Alex está entediado. Outra situação que pode acontecer é, caso apareça um oponente para desafiar o jogador, seu nível de habilidade pode não ser suficiente, o que leva à posição $A 3$, de ansiedade. Segundo o autor nenhuma das situações são experiências positivas.

Duas ações podem fazer com que Alex alcance a posição $A 4$, onde ele entra em fluxo novamente (portanto a experiência ideal). Se o garoto estiver na situação em A3, o caminho de volta ao fluxo requer que ele exercite suas habilidades, e assim chegar a A4, ou ajustar o nível de complexidade dos desafios e retroceder a A1. O autor ressalta, entretanto que o segundo caminho exemplificado não é frequentemente seguido, pois "é difícil ignorar desafios, uma vez que estamos cientes deles" Csikszentmihalyi (1990). A posição A4, segundo Csikszentmihalyi (1990) é muito mais complexa, pois exige maior capacidade cognitiva e psicomotora de Alex e, por conseguinte, é mais prazerosa.

\section{HABILIDADES DO JOGADOR}

Em todo momento, a teoria do fluxo de Csikszentmihalyi (1990) menciona o desenvolvimento de habilidades. Para fazer uma associação própria entre esse conceito e o que existe nos jogos digitais, pode-se consultar o trabalho de Aki Järvinen (2008).

Sua tese de doutorado é uma compilação de diversos métodos de análise e de projeto de jogos, desenvolvidos através de pesquisa e de sua experiência profissional. Para se chegar ao objetivo almejado nesse capítulo é necessário entender, primeiramente o que é uma habilidade do ponto de vista da ludologia (segundo Järvinen (2008), o estudo do design de jogos). O autor faz referência ao estudo de John Carroll de 1993, que buscou definir quais são as habilidades do ser humano. Järvinen (2008) faz sempre a associação dos conceitos de Carroll (1993), contextualizando-os com os jogos. Järvinen (2008) ainda menciona que a exigência dos jogos, em relação as capacidades do jogador, é natural e isso se manifesta na forma das mecânicas do jogo. Järvinen (2008) comenta que "sua execução necessita certas habilidades ou um conjunto delas, ou seja, o potencial para executar a mecânica deve ter como base habilidades cognitivas e/ou psicomotoras". Järvinen (2008) relata que, após o estudo de Carroll (1993), ele consegue traçar um panorama geral das habilidades cognitivas humanas que são comuns e pertinentes aos jogos. As habilidades tratadas são:

- Linguísticas (poder de comunicação, oratória);

- De raciocínio (poder de solucionar problemas com uso de lógica);

- De memorização (poder de lembrar informações importantes);

- De percepção visual (poder de notar sutilezas em imagens);

- De percepção auditiva (poder de notar sutilezas em sons); 
- De produção de ideias (poder de criatividade);

- Multifuncionais (que se aplicam a uma diversidade de situações);

- Físicas (força, ou velocidade acima da média);

- Psicomotoras (coordenação motora, ou destreza acima da média).

Järvinen (2008) acredita que "se soubermos melhor quais habilidades humanas os sistemas de jogos tendem a privilegiar e oferecer, nós temos uma base para um vocabulário que permita analisar e projetar experiências para jogadores".

Järvinen (2008), teoriza que existem três princípios que categorizam a qualidade das habilidades:

- Não Aplicável (n/a): habilidade que não tem relevância para o jogador. No seu estudo, Järvinen (2008) define desenvolvimento linguístico, contagem fonética, sensibilidade gramatical, produção oral, acuidade auditiva (a qualidade da audição de um indivíduo, ou seja, se ele tem audição saudável), capacidade de síntese, discriminação de sons na fala, como habilidades não aplicáveis.

- Trivial: Uma habilidade cognitiva ou psicomotora que foi desenvolvida fora da experiência de jogo, porém que tem utilidade nas suas dinâmicas. Algumas dessas habilidades, como apontadas por Järvinen (2008), podem ser compreensão verbal e textual, velocidade de leitura, aptidão ou proficiência em língua estrangeira, capacidade de discernir sons, seus tons e intensidades, sensibilidade musical, resistência à distorção de estímulos, noção do tempo, fluidez de raciocínio, de associação, de expressão.

- Não-trivial: Uma habilidade cognitiva, ou psicomotora que, segundo Järvinen (2008), é necessária para jogar um jogo e que pode ser gradativamente adquirida pelo jogador. Alguns desses exemplos mencionam que habilidades não-triviais podem ser habilidade de comunicação, estilo de pronúncia, habilidade de escrita, conhecimento léxico, compreensão de leitura especial, leitura decodificadora, habilidade de soletrar, raciocínio sequencial, indução, raciocínio quantitativo, memória associativa e visual, capacidade de visualização, raciocínio espacial, velocidade de fechamento (rapidez no reconhecimento de conceitos familiares nas formas de uma imagem, ou objeto), flexibilidade de fechamento (capacidade de reconhecer conceitos familiares em formas abstratas), velocidade perceptiva.

\section{DISCUSSÃO A LUZ DA LITERATURA}

Concluindo a revisão bibliográfica, presentes nos itens 3.1, 3.2 e 3.3, pode-se assumir que os autores estudados tangenciam uns aos outros a respeito de diversos aspectos sobre o design de jogos digitais, bem como o consumo desses e a experiência do jogador no uso desses artefatos. Nenhum deles, entretanto, faz referência aos trabalhos em paralelo. Ambos os autores Csikszentmihalyi (1990) e Järvinen (2008) escrevem sobre habilidades, o primeiro, apesar de ser do campo da psicologia, referese àquelas que são adquiridas através do tempo, mas não faz relação às suas origens, 
ou quantifica sua qualidade. Já o segundo faz um estudo aprofundado, também usando como base a psicologia, onde define três tipos de qualidades de habilidades e, mediante experimentos, averigua exemplos destas qualidades, dando respaldo para a formulação das relações.

A respeito das teorias de Hunicke et al. (2004), relacionadas a Csikszentmihalyi (1990) e Järvinen (2008), um dos tópicos mais latentes no texto é o de projeto de experiências e a previsibilidade de algo divertido e o desenvolvimento de mecânicas e dinâmicas que façam surgir comportamentos esperados pelos projetistas. Como anteriormente comentado, os três autores demonstram a intenção de entender o mesmo fenômeno, porém por perspectivas separadas. É importante ressaltar que as teorias de Järvinen (2008) não distinguem entre jogos de tabuleiro e digitais, entretanto boa parte de sua pesquisa teve videogames como objetos de estudo. Por esse motivo, o resultado desta pesquisa se aplica somente a jogos digitais - para validação dos resultados em jogos de outras mídias seriam necessários mais estudos.

O intuito deste capítulo, como previamente colocado, é fazer a junção dos três conceitos revisados. Essa junção será feita na forma de um diagrama que tem como base o esquema de Csikszentmihalyi (1990) (figura 3). O princípio da junção da teoria do fluxo com as demais está em conseguir associar seus diversos vetores aos diferentes conceitos. Começando pelo eixo Y, segundo a definição de Järvinen (2008), desafios são situações que requerem um grupo de habilidades (que podem ser aprendidas com o passar do tempo) para que sejam superados, para Hunicke et al. (2004) e Csikszentmihalyi (1990), essa declaração assemelha-se com o que define uma mecânica. Para Järvinen (2008), as condições para que um indivíduo volte ao estado de fluxo necessitam que ele, ou interaja mais com o sistema, com o intuito de adquirir novas habilidades, ou que suas habilidades já adquiridas sejam igualmente desafiadas. A experiência ideal, ou o estado de fluxo, em muito se assemelha às questões da estética que Hunicke et al. (2004) apresentam no seu trabalho. Järvinen (2008) afirma que, quando em fluxo, o indivíduo se diverte, intenção primária dos termos da estética do MDA. Em vista dessas relações, o seguinte gráfico é proposto (figura 4): 


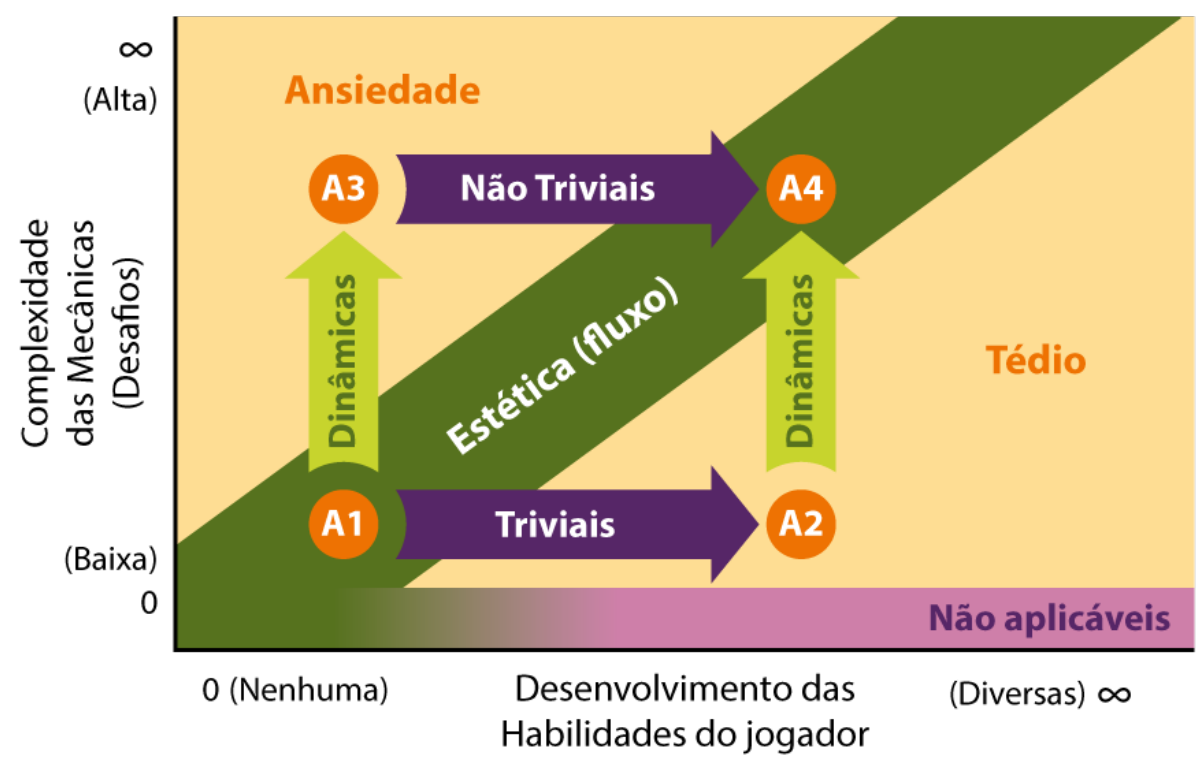

Cada par de cores nas legendas representam as teorias de cada autor:

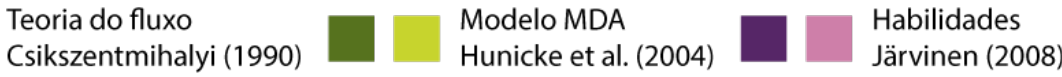

Figura 4 - Mapeamento do modelo MDA e as habilidades do jogador no gráfico da teoria do fluxo. Fonte: Elaborado pelo autor, com base na pesquisa realizada.

No gráfico (figura 4) estão relacionados o modelo MDA de design de jogos digitais (representado nas cores de tom verde), a teoria do fluxo (nas cores de tons laranja) e as habilidades do jogador (nas cores de tom roxo). Esta seleção de cores serve apenas como um guia visual para entender como as teorias estão conectadas. Como no diagrama de Csikszentmihalyi (1990), no novo esquema proposto o usuário se encontra em um estado de fluxo (A1) ao iniciar uma sessão em um jogo digital novo. Para explicar o funcionamento do gráfico, suponha-se que a sessão iniciada seja do jogo Pac-Man (Namco, 1980), durante os primeiros minutos de partida, ele se familiariza com os aspectos que compõem o jogo vivenciando a Descoberta, conceito estético de Hunicke et al. (2004), visto que ele está explorando o labirinto apresentado na tela do jogo. Ao mesmo tempo, os conceitos estéticos da Fantasia e da Submissão estão tomando conta das sensações desse indivíduo que reage emocionalmente na sua relação com o cenário do jogo. A mesma situação descrita aqui poderia ser explicada utilizando qualquer outro jogo digital como exemplo. A razão para a escolha do Pac-Man está na sua popularidade. Um fato interessante para expressar a popularidade deste jogo é que na data de seu aniversário de 30 anos de lançamento, o site de buscas Google criou uma adaptação de Pac-man e a colocou em sua página inicial. Segundo a ASTD (Associação Americana para o Treinamento e Desenvolvimento), 4.8 milhões de horas de trabalho foram gastas jogando essa adaptação naquele dia.

A medida que o jogador vai adquirindo conhecimento dos controles do jogo, bem como do funcionamento de suas mecânicas, duas situações podem acontecer. A 
primeira (A1->A3), proporcionada pelas dinâmicas, pode levar o jogador a um estado de ansiedade, devido à complexidade das mecânicas (Desafios). O jogador se sente acuado, ele acha o jogo difícil e não consegue prever suas chances de sucesso. Segundo Csikszentmihalyi (1990), esta é uma situação indesejável, entretanto Järvinen (2008) explica que, com o passar do tempo, o usuário naturalmente adquire as habilidades necessárias (não-triviais) para superar o desafio e traçar o caminho de volta ao estado de fluxo (A3->A4).

A segunda situação possível, a partir de A1 é proporcionada por habilidades triviais, ou seja, segundo Järvinen (2008), conjunto de aptidões que fazem o jogador não sentir-se desafiado, porque, por exemplo, o usuário já está acostumado a jogar Pac-man. Isso leva a experiência do jogador a percorrer o caminho A1->A2. Segundo Csikszentmihalyi (1990), mais uma vez, esta é uma situação indesejável, pois é necessário que exista um equilíbrio entre o nível de habilidade do jogador e a complexidade das mecânicas (desafio) do sistema. Para resolver essa situação - e consequentemente fazer com que o indivíduo volte a desejar jogar - as dinâmicas do jogo, ou seja, suas interações com o usuário podem tornar-se mais complexas graças a programação de uma inteligência artificial que se tora mais difícil de ser derrotada com o passar do tempo. Esta situação faz com que o jogador percorra o trajeto A2->A4, através das dinâmicas com mecânicas mais complexas que incitam uma experiência estética desafiadora.

Outra questão importante a ser ressaltada no gráfico é a presença e localização das habilidades não aplicáveis. Segundo Järvinen (2008), esse tipo de habilidade exige pouco esforço cognitivo, emocional e psicomotor e, como é de caráter básico para a interação com o sistema, pode ser considerada, quando existe baixa complexidade das mecânicas, como entediante para o jogador, visto que ele não se sentirá desafiado suficientemente. Lembrando que, segundo Csikszentmihalyi (1990), o equilíbrio de desafio e habilidade é primordial para o estado de fluxo.

\section{CONCLUSÃO}

Pelo diagrama exposto, nota-se que as diferentes teorias abordadas, apesar de não se comunicarem diretamente entre si, podem ser relacionadas sem perda de conteúdo. O modelo construído a partir da teoria do fluxo consegue contextualizar as experiências estéticas, as interações dinâmicas e as mecânicas da proposta do MDA, associando, ao mesmo tempo a teoria das habilidades do jogador. O resultado final (o gráfico) requer mais análises, assim, espera-se que outros pesquisadores possam aprofundar as associações apresentadas, ou, então, apresentar novas associações.

\section{REFERÊNCIAS}

HUNICKE, Robin; LEBLANC, Marc; ZUBEK, Robert. MDA: A formal approach to game design and game research. In: Proceedings of the AAAI Workshop on Challenges in Game Al. 2004. p. 04-04.

CSIKSZENT, Mihaly. Flow. HarperCollins, 1991.

JÄRVINEN, Aki. Games without frontiers: Theories and methods for game studies and design. 2008. 
CARROLL, John B. Human cognitive abilities. Cambridge: Cambridge University Press, 1993.

LUIZ, Rafael. Adaptação do jogo de RPG comercial Dungeons and Dragons 4th Edition para o ensino de inglês. 2011. Dissertação de Mestrado - Programa de Pós-Graduação em Design, Universidade Federal do Paraná. Curitiba, 2011.

ESA Entertainment Software Association - Disponível em http://www.theesa.com/facts/index.asp no dia 13/12/20. 